Available online on 15.05.2020 at jddtonline.info
Open Access to Pharmaceutical and Medical Research
unrestricted non-commercial use, provided the original work is properly cited

Open Access

Research Article

\title{
Preventive and Curative Effects of Atriplex Halimus L. Aqueous Extract on Benzene Provoked Hepatic Injury in Rats
}

\author{
Zeghib Khaoula ${ }^{1,2 *}$, Boutlelis Djahra Ali ${ }^{3}$ \\ 1 Department of Chemistry, Faculty of exact sciences, University of El-Oued, El-Oued 39000, El-Oued, Algeria \\ 2 University of El Oued, VTRS Laboratory, Fac. Technology, 39000 El Oued, Algeria \\ ${ }^{3}$ Department of Biology, Faculty of Natural Sciences and Life, University of El-Oued, El-Oued 39000, Algeria
}

\begin{abstract}
Objective: The present study was designed to estimate the preventive and curative potency of aqueous extract of Atriplex halimus L. (Ah) aerial parts against benzene (BZ) intoxication in liver rats.

Methods: 30 male albino rats were divided into five groups of 6 rats each: Control, $\mathrm{Ah}, \mathrm{BZ}, \mathrm{AhP}+\mathrm{BZ}, \mathrm{and} \mathrm{BZ}+\mathrm{AhC}$. BZ (100 mg/kg b.w) was added in drinking water for 15 weeks. Aqueous extract of Atriplex halimus was received intragastrically during the last 30 days of BZ exposure for curative treatment (AhC) and all the duration of BZ exposure for preventive treatment (AhP). At the end the experiment, body weight gain and relative liver weight were estimated and liver enzyme markers (AST, ALT, ALP and LDH) were analysed by spectrophotometry. Histopathological studies on hepatic tissue were also performed by the method of Hematoxylin and Eosin staining.

Results: Benzene administrated to rats caused an alteration in physiological parameters (body and organs weigh) and hepatic enzymes markers (AST, ALT, ALP and LDH). In addition, Histopathological studies showed a massive degeneration in liver tissue in BZ-exposed rats. However, treatment by Atriplex halimus especially preventive effect ameliorated most of the adverse effects induced by benzene. Ah restored the altered of physiological, biochemical and histopathological changes.
\end{abstract}

Conclusion: The present study suggests that Atriplex halimus extract could be a substantially promising hepatoprotective agent against benzene toxic effects and may be against other hepatotoxic chemical or drugs.

Keywords: Atriplex halimus, Benzene, hepatotoxicity, Rats.

Article Info: Received 12 March 2020; $\quad$ Review Completed 28 April 2020; Accepted 02 May 2020; Available online 15 May 2020

Cite this article as:

Khaoula Z, Ali BD, Preventive and Curative Effects of Atriplex Halimus L. Aqueous Extract on Benzene Provoked Hepatic

Injury in Rats, Journal of Drug Delivery and Therapeutics. 2020; 10(3):217-226

http://dx.doi.org/10.22270/jddt.v10i3.4053

*Address for Correspondence:

Zeghib Khaoula, Department of Chemistry, Faculty of exact sciences, University of El-Oued, El-Oued 39000, El-Oued, Algeria

\section{INTRODUCTION}

Liver is the most important organ responsible for xenobiotic metabolisms, acting as sensitive target site for substance modulating biotransformation. Many xenobiotics (drugs and environmental chemicals) are capable of causing some degree of liver injury 1 .

Benzene (BZ) is a ubiquitous environmental pollutant; it has been classified as a group 1A carcinogen as well as its toxic effects on the hematopoietic system are well known 2 . Benzene can also cause a great number of acute and chronic diseases, which can affect the functions of respiratory, nervous, immune, hepatic, renal, cardiovascular and reproductive systems ${ }^{3}$. Liver function as the key organ of metabolism and excretion of benzene thus, constantly endowed with the task of detoxification, inducing various disorders to the organ. Majority of benzene metabolism occurs in the liver by cytochrome p4502E1 and has genotoxic properties and play a role in benzene toxicity 4 . The general population is exposed to benzene in the atmosphere by inhalation of vapours released by motor vehicles, gasoline service stations, cigarette smoke or by ingestion of polluted foods or water 5,2 .

Herbs have been an important source of natural materials used for treatment and/or prevention of several illnesses 1. A. halimus is known in folk medicine ${ }^{6}$. This plant has been used as traditional cures for thousands of years ${ }^{7}$. In the Arab world, A. halimus was used to treat heart disease, diabetes, rheumatism ${ }^{8}$, anaemia ${ }^{9}$, and to cure stomach pains and for intestinal worms ${ }^{10}$. A. halimus is also used to treat urinary tract inflammation (cystitis) and urinary lithiasis; it accompanies any diet that requires tissue drainage and desincrustation of wastes and toxins ${ }^{11}$. In Algeria, A. halimus 
is mainly used by the local population as a remedy to treat cancer ${ }^{12}$, diabetes mellitus and anaemia ${ }^{9}$.

Given these considerations, this study was carried out to examine the possible hepatoprotective effect of $A$. halimus against benzene induced liver toxicity in rats.

\section{MATERIALS AND METHODS}

\section{Plant material}

\section{Collection and identification of Atriplex halimus $\mathrm{L}$}

Atriplex halimus L. aerial parts (stems, leaves, seeds) were collected in September 2017 from Sidi Khaled village in Biskra state, Algeria; and were identified by the botanist (Pr. Youssef Halis) at the herbarium in the Center for Scientific and Technical Research on Arid Regions (CRSTRA) of Biskra, Algeria. The plant material was washed using water, dried at room temperature, and grounded into powder and then stored at room temperature until use.

\section{Preparation of aqueous extract}

About $10 \mathrm{~g}$ of the aerial parts powder of Ah was soaked in $100 \mathrm{ml}$ of distilled water and kept at room temperature in the dark for $24 \mathrm{~h}$. Then, it was filtered through filter paper. After extraction, the solvent was removed using a rotary evaporator and incubated at $40^{\circ} \mathrm{C}$ to dry completely. The extract was weighed and stored in a refrigerator at $4^{\circ} \mathrm{C}$ for future analysis 13 .

\section{Animals}

\section{Animals and Maintenance}

A total of 48 adult male albino rats, weighing $224.98 \pm 3.81 \mathrm{~g}$, were obtained from the animal house of Pasteur Institute, Algeria. They were placed and kept in animal house of Molecular and Cellular Biology Department, University of ElOued, Algeria. Animals were adapted for 2 weeks under the same laboratory conditions of photoperiod $(12 \mathrm{~h}$ light/12 $\mathrm{h}$ dark) with relative humidity $64 \pm 2 \%$ and room temperature of $19 \pm 1^{\circ} \mathrm{C}$. Standard rat food and tap water were available ad libitum for the duration of the experiments.

\section{Experimental design}

The adult Wistar albino rats were randomly divided into eight groups, each containing 6 rats as follow: Groups 1: was serving as a control and received normal water; Groups 2: was treated orally by gavage $200 \mathrm{mg} / \mathrm{kg}$, b.w (3 days/week) of aqueous extract of Atriplex halimus L (Ah) for 15 weeks; Groups 3: received in drinking water $100 \mathrm{mg} / \mathrm{kg}$, b.w/day of benzene (BZ) daily for 15 weeks; Group 4: was concomitantly administered preventively Ah $200 \mathrm{mg} / \mathrm{kg}, 3$ days/week) with BZ for 15 weeks (AhP+BZ); Group 5: was firstly treated by BZ only for 11 weeks then treated curatively by $300 \mathrm{mg} / \mathrm{kg}$, b.w/day of $\mathrm{Ah}(\mathrm{BZ}+\mathrm{AhC})$ for 30 days. Body weight was recorded periodically during the experiment weeks.

\section{Samples preparation}

At the end of treatment, rats were fasted for $16 \mathrm{~h}$, anaesthetized with chloroform by inhalation, and then decapitated. The blood samples were transferred into serum separating tubes and prepared by centrifugation for $10 \mathrm{~min}$ at 3000 revolutions/min and it was utilized for biochemical parameters analysis.

We made a longitudinal abdominal opening in animals and excise the liver. After removing all connective tissue from the organs, the liver were weighed, and fixed in formaldehyde (10\%) for histopathological examination.

\section{Biochemical parameters analysis}

ALT, AST, ALP, and LDH activities were measured by kinetic colorimetric method using technical cards and commercial kits obtained from Spinreact and BioSystems (Barcelona, Spain).

\section{Histopathological study}

After rats sacrificed, the parts of studied tissues were removed and immersed in fixative (10\% formaldehyde) until the time of slices preparation. The samples were dehydrated in ascending graded series of ethanol, cleaned with toluene, and immersed in paraffin. Sections of $4 \sim 6 \mathrm{~mm}$ were prepared from paraffin blocks using a Histoline Rotary Microtome, and coloured with hematoxylin and eosin. Histopathological evaluation was performed with a light microscope.

\section{Statistical analysis}

Data was expressed as mean \pm standard deviation $(\mathrm{M} \pm \mathrm{SD}$ ) of six animals. Statistical analysis was carried out by using Student T-test to compare means between two groups. Results were evaluated using the Minitab and EXCEL software. Differences were considered significant at $\mathrm{p} \leq 0.05$.

\section{RESULTS}

\section{Body weight gain and relative liver weight}

$\mathrm{BZ}$ exposure to rats caused a decrease in body weight gain $(p<0.001)$ and in relative liver weight $(p<0.001)$ compared to the control rats. Preventive Ah treatment of $\mathrm{BZ}$ exposure rats $(\mathrm{AhP}+\mathrm{BZ})$ was restored the body weight gain $(\mathrm{p}<0.01)$, relative liver weight $(\mathrm{p}<0.01)$ as compared to $B Z$ rats. Curative treatment of the aqueous extract of Ah was significantly $(\mathrm{p}<0.05)$ increase body weight gain and relative liver weight compared to BZ rats (Table 1 ).

Table 1: Initial body weight, body weight gain, and relative liver weight in control and experimental groups.

\begin{tabular}{|l|c|c|c|}
\hline Parameters & Initial body weight (g) & Body weight gain (g/d) & Relative liver weight (g/100 g bw) \\
\hline Control & $221.17 \pm 7.73$ & $0.74 \pm 0.10$ & $2.340 \pm 0.088$ \\
\hline Ah & $233 \pm 4.71$ & $0.719 \pm 0.064$ & $2.319 \pm 0.052$ \\
\hline BZ & $234.2 \pm 12.1$ & $0.38 \pm 0.031^{* * *}$ & $2.097 \pm 0.033^{* * *}$ \\
\hline AhP+BZ & $219.25 \pm 7.16$ & $0.76 \pm 0.072^{\mathrm{b}}$ & $2.216 \pm 0.033^{* * \mathrm{~b}}$ \\
\hline BZ+AhC & $229.25 \pm 6.34$ & $0.66 \pm 0.037^{\mathrm{b}}$ & $2.291 \pm 0.085^{\mathrm{a}}$ \\
\hline
\end{tabular}




\section{Biochemical parameters}

Results presented in Table 2 showed a significant decrease in liver ALT $(\mathrm{P}<0.001)$ and serum ALP $(\mathrm{P}<0.05)$ activities, and non-significant change in liver AST and serum LDH activities in benzene-intoxicated group when compared with the control group. However, treatment with aqueous extract of $A$. halimus extract along with benzene provided protection to these enzymes activities. Atriplex curative treatment attenuated the decrease of ALT $(\mathrm{P}<0.001)$ and the increase of LDH activities but did not affect the serum ALP level when compared with benzene group. Treatment with Ah alone decreased serum LDH activity only.

Table 2: Liver and serum enzymes activities in the control and experimental groups.

\begin{tabular}{|l|c|c|c|c|}
\hline Parameters & Liver ALT (U/I) & Liver AST (U/l) & Serum ALP (U/l) & Serum LDH (U/I) \\
\hline Control & $219.9 \pm 11.5$ & $434.1 \pm 46.1$ & $343.5 \pm 16.5$ & $3166 \pm 149$ \\
\hline Ah & $206.5 \pm 8.54$ & $422.8 \pm 45.9$ & $319.6 \pm 19.7$ & $2227 \pm 135^{* *}$ \\
\hline BZ & $144.68 \pm 9.06^{* * *}$ & $472.8 \pm 32.4$ & $234.0 \pm 23.6^{*}$ & $3324+216$ \\
\hline AhP+BZ & $188.8 \pm 12.2^{* b}$ & $404.3 \pm 33.1$ & $371.5 \pm 29.1^{\mathrm{c}}$ & $2831+187$ a \\
\hline BZ+AhC & $201.1 \pm 16.1^{\mathrm{b}}$ & $550.20 \pm 8.83^{* * *} \mathrm{c}$ & $222.6 \pm 29.7^{*}$ & $3098+118^{\mathrm{a}}$ \\
\hline
\end{tabular}

\section{Histopathological study}

The light micrographs of liver tissues demonstrated normal architecture of hepatic cells with normal central and portal area in the control and Ah groups (Figure 1,2), while BZintoxicated rats revealed severe pathological changes manifested by degeneration and dilatation of the central vein, hepatic cell necrosis, congestion and dilatation of hepatic sinusoids, and accumulation of inflammatory cells around central vein. Moreover, portal areas showed fibrous expansion of most portal areas, severe infiltration of portal tract areas with inflammatory cells, congestion and dilatation of the portal vein and oedema (Figure 3). However, livers of BZ rats treated with $A$. halimus extract markedly reduced and attenuated the histological alterations (Figure 4). Moderate congestion and dilatation of the central and portal vein and mild inflammation was showed in these groups (Figure 5).

Histopathological changes are graded and summarized in Table 3. Histological grading was made according to four severity grades: - (none); + (mild); ++ (moderate) and +++ (severe).
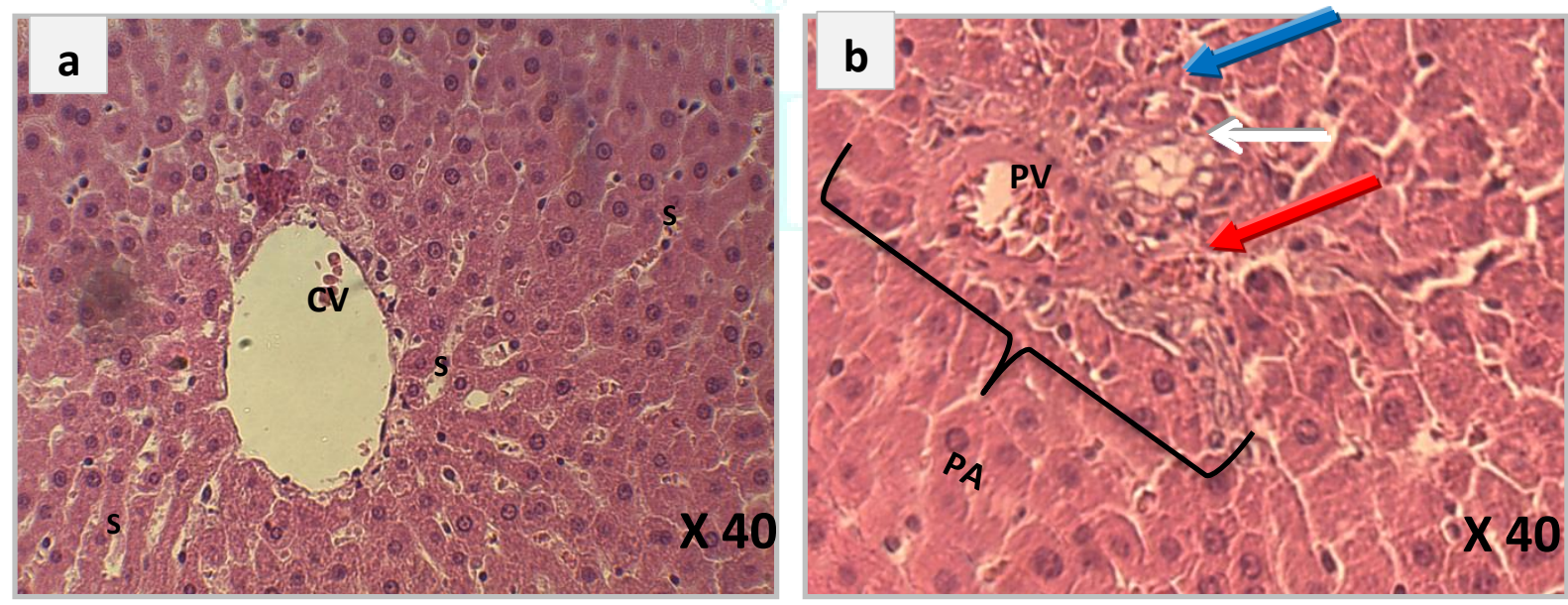

Figure 1: Liver section from control group showing normal structural morphology in (a) the central area, sinusoids (S) and central vein (CV) and (b) normal portal area including portal vein (PV), bile duct (blue arrow), artery (white arrow) and portal tract (red arrow). 

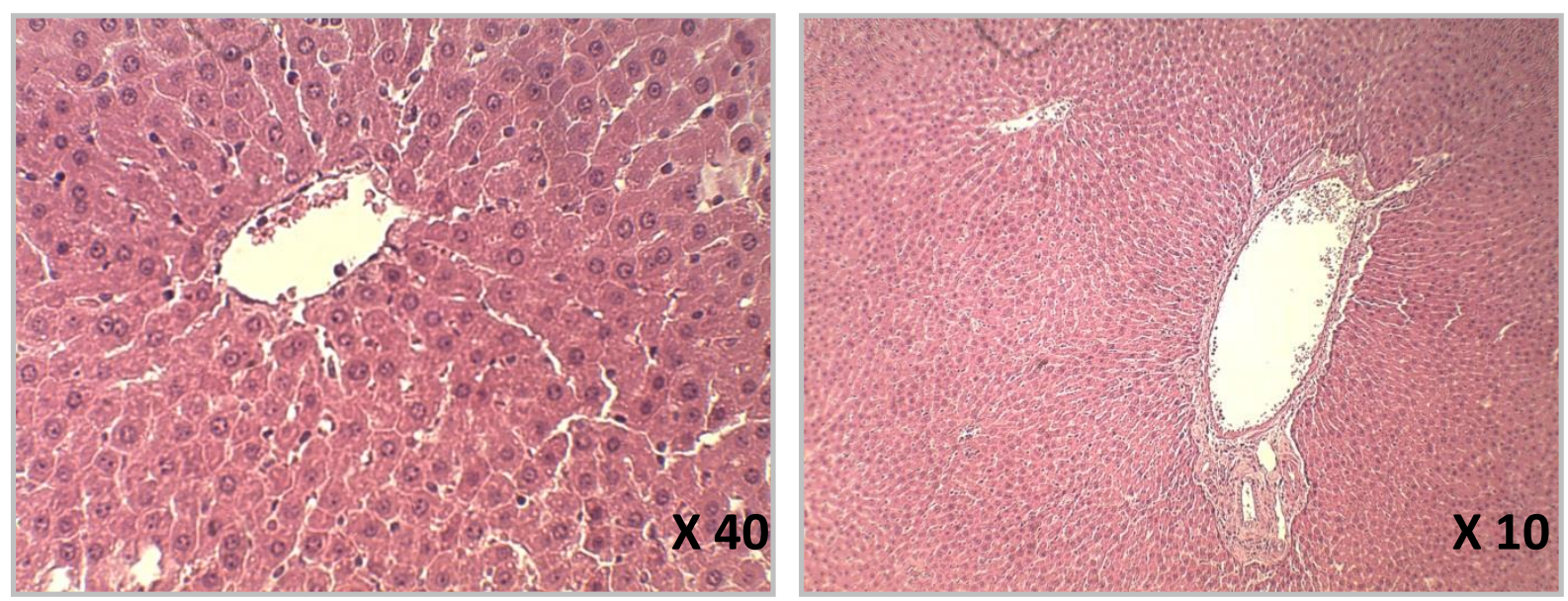

Figure 2: Liver section from Ah group showing normal architecture.
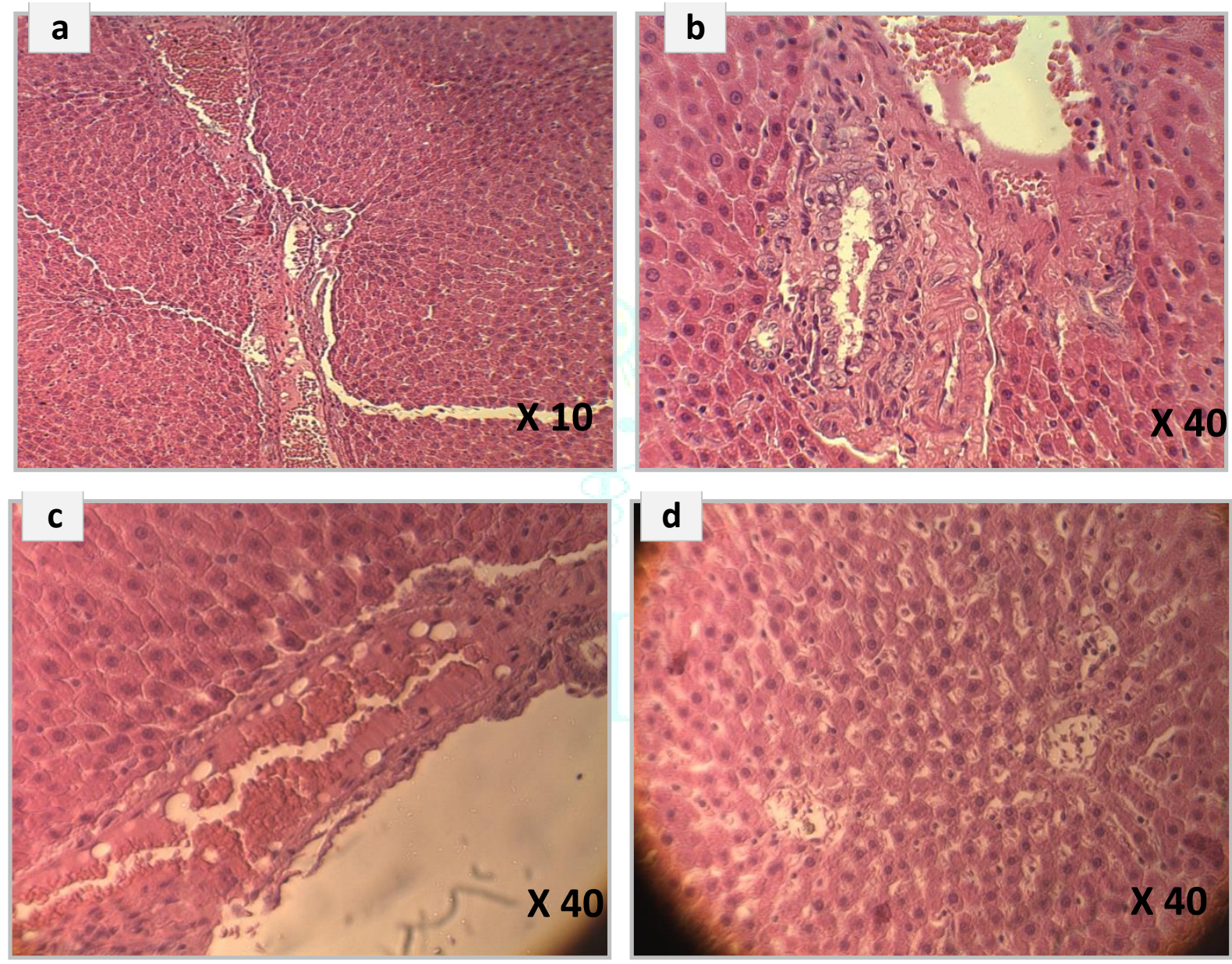

Figure 3: Liver section from BZ group showing: (a) fibrosis, congestion, (b) fibrosis and lymphocytic inflammation in portal area, (c) lymphocytic inflammation, congestion and edema in central vein (d) sinusoidal congestion and dilatation, cytoplasmic vacuolation. 

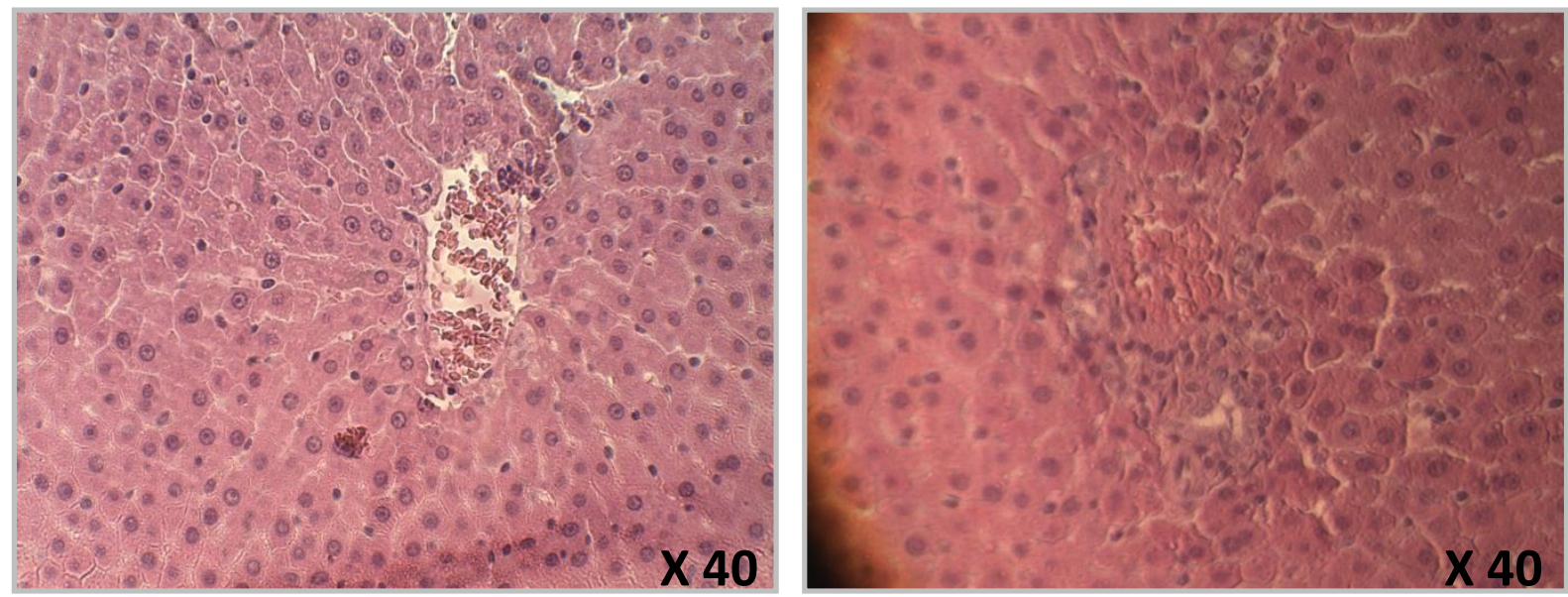

Figure 4: Liver section from $\mathrm{AhP}+\mathrm{BZ}$ group showing mild degeneration in central and portal areas.
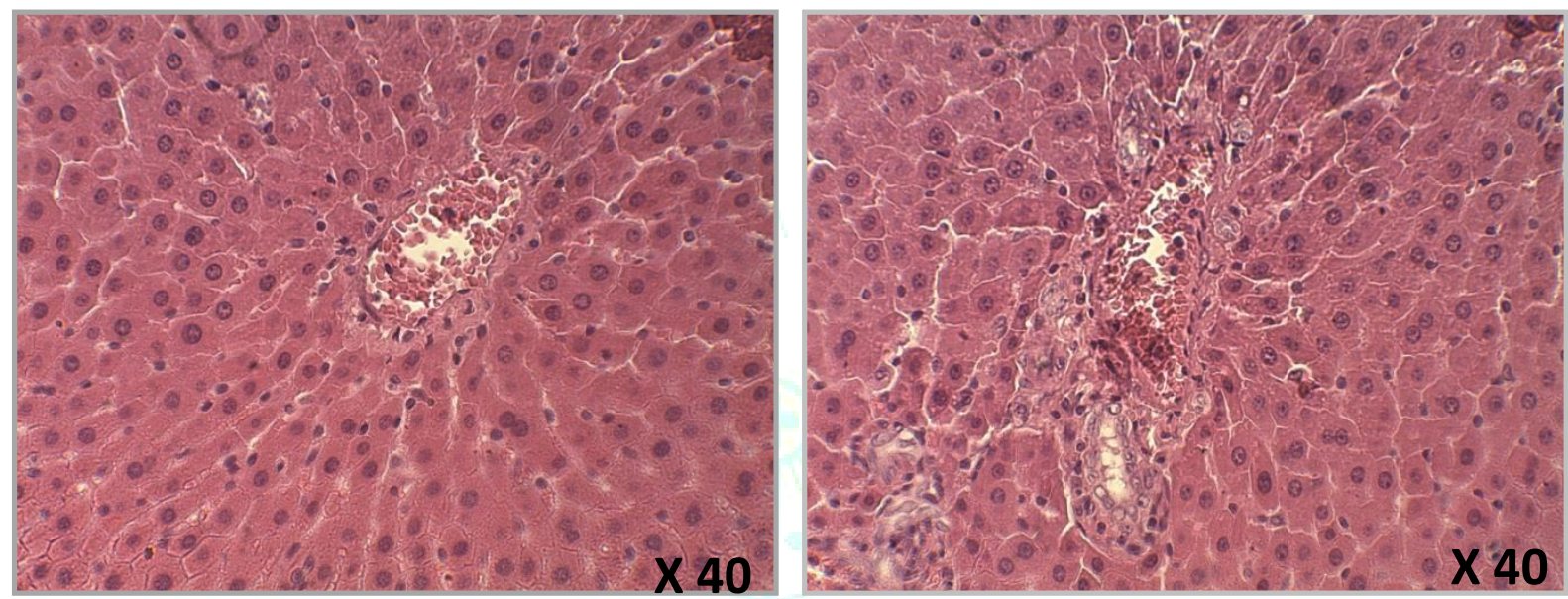

Figure 5: Liver section from BZ+AhC group showing: a moderate degree of liver damage.

Table 3: Semi-quantitative recording of architectural damage on histopathological analysis of the liver of control and treated rats

\begin{tabular}{|c|c|c|c|c|c|}
\hline Parameters & Control & $\mathbf{A h}$ & $\mathbf{B Z}$ & AhP+BZ & BZ+AhC \\
\hline Portal fibrosis & - & - & +++ & - & - \\
\hline Inflammatory infiltration & - & - & ++++ & + & +++ \\
\hline Hepatic necrosis & - & - & ++ & - & ++ \\
\hline Congestion and dilatation of sinusoids & - & - & ++ & - & - \\
\hline Hepatic vein dilatation and congestion & - & - & ++++ & + & + \\
\hline Cytoplasmic vacuolations & - & - & ++ & + & - \\
\hline Expansion of portal tract & - & - & ++++ & - & ++ \\
\hline
\end{tabular}

\section{DISCUSSION}

Nutritional therapy combined with natural agents from food and herbs have been proposed as the optimal management for liver disease ${ }^{14}$. In order to search new drug contained in plants, characterized by effective treatment of different diseases and fewer side effects, it is necessary to carry out pharmacological studies on plants used by traditional healers. In this study, we evaluate the possible pharmacological effects of $A$. halimus against hepatotoxicity induced by benzene.
Decreased body weight is an important indicator for assessing the deterioration of health status ${ }^{15}$. In our study, $\mathrm{BZ}$ exposure at a dose $(100 \mathrm{mg} / \mathrm{kg})$ resulted in a significant decrease in body weight and relative liver weight. In agreement with Ibrahim et al. (2011) 16 who reported a significant decline in the body weight gain of the animals intoxicated with BZ, suggesting that reduced of the body weights may be due to the overall increased degradation of lipids and proteins as a result of the direct effects of BZ.

In contrast, treatment by Atriplex especially preventively with $\mathrm{BZ}$ restored the weight of the body, liver near to 
normal, which can indicate the beneficial effect of Ah against BZ as evidenced macroscopically.

Liver function was assessed by examining the activities of AST, ALT, ALP, and LDH in rats. The results demonstrated that the activities of liver ALT and serum ALP were reduced after benzene intoxication. Our data were consistent with Adeyemi et al. (2009) ${ }^{[17}$ who reported a reduction in liver ALT, AST activities and elevation on serum LDH activity of rats exposed to benzene. Also, the study of Hegazy and Kamel, (2014) 18 showed that occupational exposure to benzene affected four important hepatic marker enzymes activities (LDH, ALP, ALT and AST) in serum.

As a reason for our observation, liver enzymes ALT, AST, and ALP are known markers enzymes for the assessment of the functional integrity of the liver cells. The decrease of liver ALT activities after BZ exposure may be due to the leakage of this enzyme from the liver cytosol into the blood stream ${ }^{19}$. Liver cell membranes were damaged by benzene via the generation of ROS, leading to lipid peroxidation of biomembrane causing leakage of cellular components 20 . Alkaline phosphatase is a marker enzyme for plasma membrane; two abnormal levels of alkaline phosphatase have been proposed: increase or decrease in normal levels. Damage to biological membrane may lead to any of the two abnormalities 17. Lactate dehydrogenase is an important enzyme in biological processes; it is considered as specific indicators for hepatic dysfunction and damage 21 .

On the other hand, our results show that the treatment of rats by $A$. halimus significantly restored the liver enzymatic activity, thus indicating that Atriplex inhibits liver damage caused by BZ.

Histopathological observation of liver tissue sections from the BZ group confirmed the biochemical results and showed clear signs of hepatotoxicity. Stained sections reveal severe degeneration in liver tissue, large damage of central and portal liver areas, fibrosis, inflammatory cell infiltration, vascular congestion, vacuolization, and dilatation. Overproduction of ROS in chronic BZ exposure can cause damage of important biomolecules (proteins, lipids, and DNA) and increase intra-tissular pro-inflammatory and fibrogenic factors, which can lead to cell death (necrotic) and chronic inflammation associated with renal and hepatic tissues damage 22. However, Atriplex improved the hepatic and renal histological changes induced by BZ. The histoprotective effect of $\mathrm{Ah}$ is due to the presence of antioxidant and anti-inflammatory components in this plant.

\section{CONCLUSION}

In light of the results obtained, the present study affirmed a beneficial ability of $A$. halimus aqueous extract to prevent and reduce $\mathrm{BZ}$-induced liver damage. It also reveals that $A$. halimus is a robust medicinal herb for developing as a phytomedicine against hepatic disorders. Hence, dietary supplementation with $\mathrm{Ah}$ could be a useful method to protect populations at high risk of BZ chronic intoxication.

\section{Acknowledgements}

This work was supported by Faculty of Sciences of Nature and Life, University of El-Oued, Algeria. The first author is grateful to Dr. Derouiche Samir, head of department of cellular and molecular biology and lecturer at the Faculty of Natural and Life Sciences, University of El-Oued, for their constant support and guidance throughout the study.

\section{Conflicts of interest}

The authors report that no conflict of interest

\section{REFERENCES}

1. Abdou RH, Saleh SY, Khalil WF. Toxicological and biochemical studies on Schinus terebinthifolius concerning its curative and hepatoprotective effects against carbon tetrachloride-induced liver injury. Pharmacognosy magazine, 2015; 11(Suppl 1):S93.

2. Falzone L, Marconi A, Loreto C, Franco S, Spandidos DA, Libra M. Occupational exposure to carcinogens: Benzene, pesticides and fibers. Molecular medicine reports, 2016; 14(5):4467-74.

3. D'Andrea, M. A., \& Reddy, G. K. Health risks associated with benzene exposure in children: a systematic review. Global pediatric health, 2018; 5:2333794X18789275.

4. Uzma N, Kumar BS, Hazari MA. Exposure to benzene induces oxidative stress, alters the immune response and expression of p53 in gasoline filling workers. American journal of industrial medicine, 2010; 53(12):1264-70.

5. Wilbur SB, Keith S, Faroon 0, Wohlers D. Toxicological profile for benzene. 2007.

6. Kabbash, A., \& Shoeib, N. Chemical and biological investigation of some secondary metabolites in Atriplex halimus growing in Egypt. Natural product communications, 2012; 7(11):1934578X1200701114.

7. Walker, D. J., Lutts, S., Sánchez-García, M., \& Correal, E.. Atriplex halimus L.: Its biology and uses. Journal of Arid Environments, 2014; 100:111-121.

8. Parvez MK, Arbab AH, Al-Dosari MS, Al-Rehaily AJ, Alam P, Ibrahim KE, et al. Protective effect of Atriplex suberecta extract against oxidative and apoptotic hepatotoxicity. Experimental and therapeutic medicine, 2018; 15(4):3883-9.

9. Mohammedi Z. Resistance, pharmacology properties and nutritional value of a shrub from arid environments Atriplex halimus. Res J Med Plant, 2016; 10:10-8

10. Slama K, Boumendjel M, Taibi F, Boumendjel A, Messarah M. Atriplex halimus aqueous extract abrogates carbon tetrachloride-induced hepatotoxicity by modulating biochemical and histological changes in rats. Archives of physiology and biochemistry, 2018:1-2.

11. Nedjimi B, Guit B, Toumi M, Beladel B, Akam A, Daoud Y. Atriplex halimus subsp. schweinfurthii (Chenopodiaceae): description and ecology, use as forage and as a therapeutic plant. Fourrages, 2013; (216):333-8.

12. Alves-Silva JM, Romane A, Efferth T, Salgueiro L. North African medicinal plants traditionally used in cancer therapy. Frontiers in pharmacology, 2017; 8:383.

13. Murugan R, Parimelazhagan T. Comparative evaluation of different extraction methods for antioxidant and anti-inflammatory properties from Osbeckia parvifolia Arn.-An in vitro approach. Journal of King Saud University-Science, 2014; 26(4):267-75.

14. Zulkawi N., Ng K. H., Zamberi R., Yeap S. K., Jaganath I. B., Satharasinghe D., \& Alitheen N. B. The in vivo hepato-recovery effects of the polyphenolrich fermented food Xeniji ${ }^{\mathrm{TM}}$ on ethanol-induced liver damage. RSC Advances, 2017; 7(61):38287-38299.

15. Ullman-Culleré M. H., \& Foltz C. J. Body condition scoring: a rapid and accurate method for assessing health status in mice. Comparative Medicine, 1999; 49(3):319-323.

16. Ibrahim KS, Saleh ZA, Farrag AR, Shaban EE. Protective effects of zinc and selenium against benzene toxicity in rats. Toxicology and industrial health, 2011; 27(6):537-45.

17. Adeyemi, O., Ajayi, J. O., Olajuyin, A. M., Oloyede, O. B., Oladiji, A. T., Oluba, O. M., ... \& Adebayo, E. A. Toxicological evaluation of the effect of water contaminated with lead, phenol and benzene on liver, kidney and colon of Albino rats. Food and chemical toxicology, 2009; 47 (4):885-887.

18. Hegazy R. M., \& Kamel H. F. Oxidant Hepatic \&/or Haem. Injury on FuelStation Workers Exposed to Benzene Vapor, Possible Protection of Antioxidants. American Journal of Medicine and Medical Sciences, 2014; 4(2):34-46.

19. Çavuşoğlu K., Yalçın E., Yapar K., Oruç E., Gür B., \& Çiçek F. The effects of grape seed extract against toxicity of benzene on liver and kidney tissues of albino mice: biochemical evaluation.Turkish Journal of Biochemistry, 2015; 40(1):66-73.

20. El-Shakour A. A., El-Ebiare A. S., Ibrahim Y. H., Abdel M. A. E., \& ElMekawy A. M. Effect of benzene on oxidative stress and the functions of liver and kidney in rats. J Environ Occup Sci, 2015; 4:34-9.

21. Dere E., \& Ari F. Effect of benzene on liver functions in rats (Rattus norvegicus). Environmental monitoring and assessment, 2009; 154(14):23-27.

22. Cottone S., Lorito M. C., Riccobene R., Nardi E., Mule G., Buscemi S., ... \& Cerasola G. Oxidative stress, inflammation and cardiovascular disease in chronic renal failure. Journal of nephrology, 2008; 21(2):175-179. 